\title{
Angiographic Complete versus Clinical Selective Incomplete Percutaneous Revascularization in Heart Failure Patients with Multivessel Coronary Disease
}

\author{
Chieh-Yu Chang, Chun-Chi Chen, I-Chang Hsieh, Ming-Jer Hsieh (D), Cheng-Hung Lee, \\ Dong-Yi Chen, Ming-Lung Tsai, Ming-Yun Ho, Jih-Kai Yeh, Yu-Chang Huang, \\ Yu-Ying Lu, Chao-Yung Wang, Shang-Hung Chang, and Ming-Shien Wen \\ Division of Cardiology, Department of Internal Medicine, Chang Gung Memorial Hospital, \\ Chang Gung University College of Medicine, Address: No. 5, Fu-Hsing Street, Kwei-Shan, Taoyuan, Taiwan \\ Correspondence should be addressed to Ming-Jer Hsieh; mingjer.hsieh@gmail.com
}

Received 27 March 2020; Revised 3 June 2020; Accepted 24 June 2020; Published 27 July 2020

Academic Editor: Yuichiro Maekawa

Copyright $\odot 2020$ Chieh-Yu Chang et al. This is an open access article distributed under the Creative Commons Attribution License, which permits unrestricted use, distribution, and reproduction in any medium, provided the original work is properly cited.

\begin{abstract}
Background. Patients with multivessel disease (MVD) often pursue complete revascularization (CR) during percutaneous coronary intervention (PCI) to improve prognosis. However, angiographic CR is not always feasible and is associated with some procedure-related complications in heart failure (HF) patients with MVD. Clinical selective incomplete revascularization (IR) may be reasonable for these high-risk patients, but its role in long-term outcomes remains uncertain. Methods. Six hundred patients with HF and MVD submitted to PCI were enrolled. Major adverse cardiac events (MACEs) were defined as a composite of recurrent myocardial infarction, any revascularization, and all-cause mortality at 5 years. Results. During a mean follow-up period of 3.7 \pm 1.9 years, there was no significant difference in 5-year MACEs between selective IR and successful angiographic CR in HF patients with MVD. However, patients who failed CR had a significantly greater incidence of 5-year MACEs than those in the other two groups (failed CR: $46.4 \%$ vs. selective IR: $27.7 \%$ vs. successful CR: $27.8 \%, p<0.001$ ). Conclusions. Long-term outcomes of selective IR were comparable with those of successful angiographic CR in HF patients with MVD. However, patients that failed CR showed 2.53-fold increased risk of MACEs compared to patients undergoing either selective IR or successful angiographic CR. A more comprehensive planning strategy should be devised before PCI in HF patients with MVD.
\end{abstract}

\section{Introduction}

Heart failure (HF) owing to ischemic cardiomyopathy is currently an epidemic and an increasing health care burden due to high mortality and poor prognosis $[1,2]$. Guidelinedirected medical therapy (GDMT), which includes the use of angiotensin-converting enzyme inhibitors (ACEi) or angiotensin receptor blockers (ARB), beta-blockers, and mineralocorticoid receptor antagonists (MRA), has greatly improved the survival rate of patients with reduced ejection fraction (EF) [2]. Moreover, compared with GDMT alone, GDMT plus revascularization strategies might further improve the clinical outcome in HF patients with ischemic cardiomyopathy [3].
Multivessel disease (MVD) has been found in nearly half of the patients with coronary artery disease (CAD) [4-6]. Traditionally, clinical guidelines recommend coronary artery bypass grafting (CABG) as the first choice for MVD revascularization [7]. Randomized controlled trials conducted for specific scenarios, such as left main disease and low anatomic complexity, showed that percutaneous coronary intervention (PCI) is not inferior to CABG in reduction of major adverse cardiovascular events (MACEs) [8-10]. In addition, because of the advances in procedural techniques, devices, and operator experiences, PCI has become an alternative choice in the management of MVD patients with high surgical risk, such as those with HF [11]. 
As extensive revascularization is the main advantage of CABG over PCI, it is reasonable to pursue angiographic complete revascularization (CR) rather than culprit-only or incomplete revascularization (IR) in patients undergoing PCI instead of CABG [12]. However, angiographic CR cannot always be achieved in daily practice. A reasonable IR that is guided by anatomic, functional, and physiological parameters identifying small myocardial area at risk may be another choice [13]. However, data comparison between reasonable IR and CR in HF patients with MVD was scarce.

In this real-world prospective registry, we retrospectively analyzed outcomes in HF patients with MVD who underwent PCI either with angiographic successful CR or with residual coronary stenosis. Furthermore, we found patients with residual coronary stenosis, which may result from either initial selective IR based on clinical evidence of nonviable tissue or failed $\mathrm{CR}$ with clinical evidence of viable tissue. Therefore, the goal of this study was comparison of the 5-year outcomes of different PCI revascularization strategies with either successful CR, selective IR, or failed CR in HF patients with MVD.

\section{Materials and Methods}

2.1. Study Population, Definitions, and Clinical Follow-Up. In the present study, we enrolled HF patients with MVD who received PCI between April 2000 and February 2017 from the Cardiovascular Atherosclerosis and Percutaneous TrAnsluminal INterventions (CAPTAIN) registry [14, 15]. This study is designed retrospectively by using a single center registry database which recorded all clinical parameters prospectively. Patients with evidence of left ventricular EF $<40 \%$ and stenosis $>50 \%$ in at least two native coronary arteries were included. Patients who underwent bypass surgery, showed inability to tolerate dual antiplatelet therapy, and were unable to follow the study protocol were excluded. Dual antiplatelet therapy, which combined aspirin with a P2Y12 inhibitor (clopidogrel or ticagrelor), was administered to all enrolled patients for at least 9 months according to national health insurance regulations. This study complied with the Declaration of Helsinki and ethical approval for this study was obtained from the Institutional Review Board of Chang Gung Medical Foundation. All participants provided informed consent for PCI and the follow-up protocol.

As the study flowchart in Figure 1, we finally categorized MVD patients in this study into three groups: CR, selective IR, and failed CR. Angiographic MVD was defined as the presence of $\geq 50 \%$ stenosis in at least two major coronary vessels or their major branches. Because the smallest stent diameter in our laboratory was $2.25 \mathrm{~mm}$, the definitions of $\mathrm{CR}$ and IR in previous studies were modified as follows [16-18]. All patients in angiographic CR group were those without angiographic residual $\geq 50 \%$ stenosis in any coronary arteries with $>2.25 \mathrm{~mm}$ in diameter regardless of viability test. Each patient in selective IR group had both viable and non-viable myocardium. Treating stenotic vessels supplying possible viable tissue and sparing stenotic vessel supplying non-viable tissue by direct or indirect viability test were performed in patients in selective IR group. Failed CR were defined as angiographic residual stenosis with viable myocardium that received failed stenotic vascular intervention. Myocardium with one of the following conditions was considered as nonviable tissue: $Q$ wave in previous electrocardiogram without new ST-T changes [19]; thinning $<5 \mathrm{~mm}$, akinetic myocardial wall with left ventricular endsystolic volume $>130 \mathrm{~mL}$ in echocardiography [20-22]; and infarction without viability detected on stress myocardial perfusion scan [22]. Myocardium with one of the following conditions was considered as viable tissue: dynamic ST-T changes in electrocardiogram [19]; left ventricular end-diastolic wall thickness $\geq 5 \mathrm{~mm}$ without features indicating non-viability in echocardiography $[20,22]$; and ischemia with viability detected on stress myocardial perfusion scan [22]. Long-term MACEs during follow-up were defined as a composite of recurrent myocardial infarction (MI), any revascularization, and all-cause mortality in 5 years. Recurrent MI was diagnosed in cases with prolonged chest pain that lasted for more than 30 minutes, ST segment elevation or depression of at least $0.2 \mathrm{mV}$ in two or more contiguous electrocardiogram leads, and significantly elevated levels of cardiac enzymes. Any revascularization was defined as further PCI or CABG after discharge from index hospitalization due to any clinical reason.

A review of all patients' medical records was conducted to obtain information on clinical status, medical management, and occurrence of any adverse event. Patients were followed up clinically in the outpatient department or through phone calls. Follow-up was scheduled at 1, 2, and 3 months after the procedure and every 3 months thereafter. The index date was defined as the date that patients underwent final PCI. Baseline characteristics were defined as the data from discharge or outpatient clinic diagnosis before the index date. Patients were followed until the first occurrence of any event, including MI, revascularization, and death after their index date for 5 years or until they completed uneventful follow-up until November 30, 2019.

2.2. Statistical Analysis. All results are presented as means \pm standard deviation or percentages and categorical data are presented as numbers. The normality of all variables was analyzed. For continuous data, groups were compared using the $t$ test or Wilcoxon rank-sum test based on the distribution. Categorical variables were compared using the chi-squared test. The clinical outcomes were compared between angiographic CR, selective IR, and failed CR groups. A multivariate Cox proportional hazards regression model was used to evaluate outcomes in patients with different revascularization strategies. Baseline characteristics and variables with $p$ value $<0.2$ in comparison between these three groups were adjusted in Cox proportional hazards regression analysis. To eliminate the procedure related short term effect, landmark survival analysis (index to 30 days, and 30 days to 5 years) was performed. Multivariate analysis was performed using the Cox regression model to identify independent predictors for 5-year MACEs. All results with $p$ value less than 0.05 


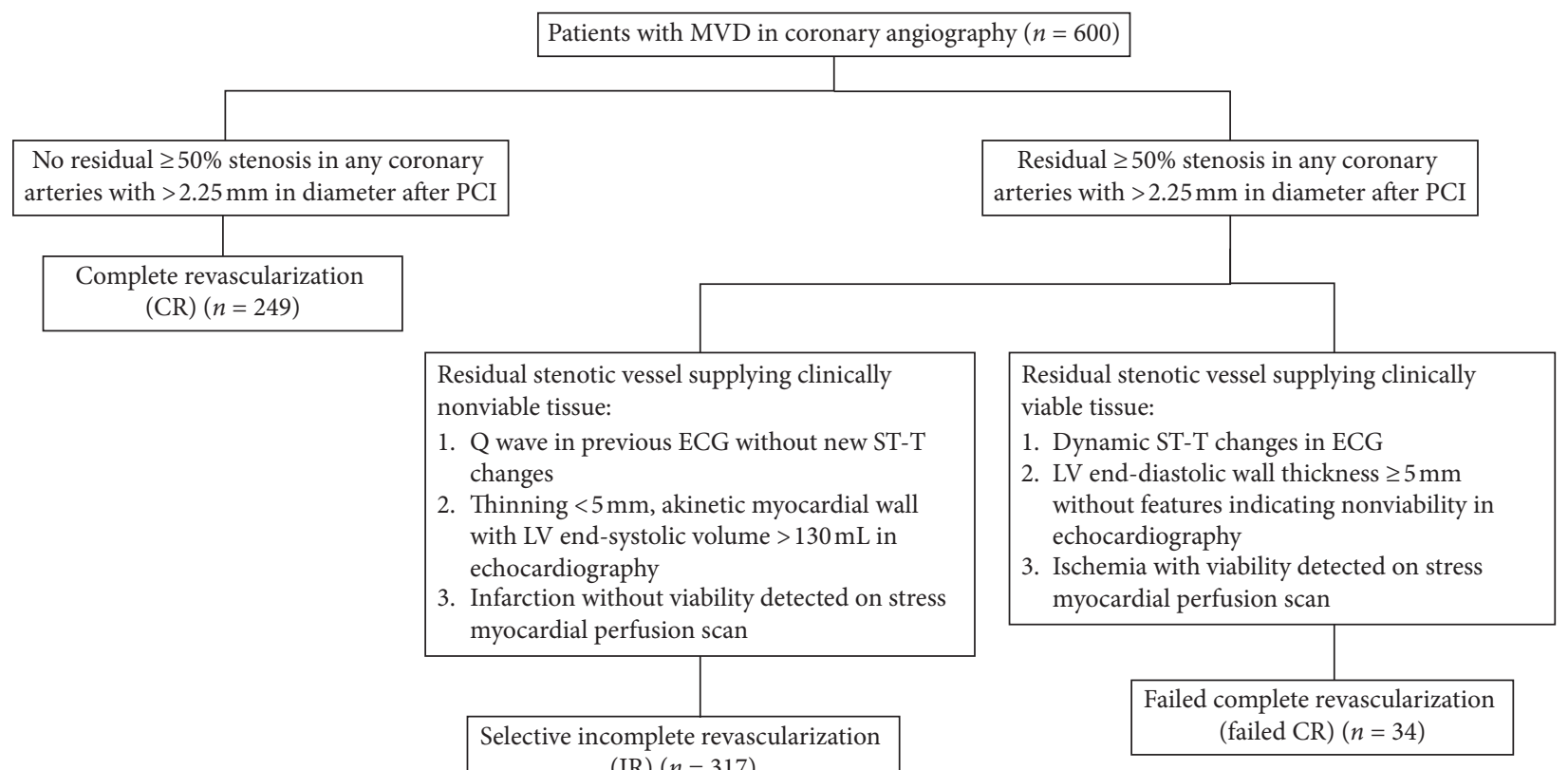

Figure 1: Study flow chart.

were defined as significant. Survival was investigated using the log-rank test with Kaplan-Meier curves. All statistical analyses were performed using SPSS 17.0 for Windows.

\section{Results}

3.1. Patient Characteristics. A total of $600 \mathrm{HF}$ patients with MVD, including 249 patients with successful angiographic CR and 351 patients receiving PCI with residual coronary stenosis, were enrolled for further analysis. In patients with residual coronary stenosis, 317 patients underwent PCI with initial selective IR and 34 patients attempted CR initially but eventually failed PCI. The successful rate of angiographic CR was $88 \%$. The reasons of failed CR included chronic total occlusion (CTO) lesion wiring failure (38.2\%), inability of patient hemodynamic condition to tolerate the whole PCI procedure (35.4\%), presence of an un-dilatable lesion (17.6\%), and occurrence of procedure related complications (8.8\%).

Baseline characteristics of the study population are presented in Table 1. In general, there was no significant difference between angiographic CR and selective IR in all clinical variables including age, sex, diabetes mellitus, hypertension, hyperlipidemia, smoking, family history of CAD, previous MI, previous stroke, acute coronary syndrome presentation, NYHA functional class, LVEF, estimated glomerular filtration rate, chronic kidney disease stage, calcified lesion, ostial lesion, bifurcation lesion, CTO lesion, use of drug-eluting stents, and use of long-term GDMT including ACEi/ARB therapy, beta-blocker therapy, and MRA. Most incidences of clinical variables in failed CR group were also similar to those in the other two groups, but patients in failed CR group had significant higher incidence of NYHA functional class 4, lower LVEF, and more CTO lesions, compared with those in angiographic CR and selective IR groups.
3.2. Clinical Outcomes between Successful Angiographic CR, Selective IR, and Failed CR. After a mean follow-up duration of $3.7 \pm 1.9$ years, 187 patients (31.2\%) suffered from 5 -year MACEs. Kaplan-Meier analysis demonstrated no difference of MACEs between angiographic CR and selective IR. A significant higher incidence of 30-day MACEs with failed CR was noted (30-day MACEs in angiographic CR, selective IR, and failed $\mathrm{CR}=4.4 \%, 3.2 \%$, and $17.6 \%$; log-rank $p<0.001)$. Even when excluding 30-day MACEs after PCI, failed CR still had higher incidence of MACEs from 30 days to 5 years (30-day to 5-year MACEs in angiographic CR, selective IR, and failed $\mathrm{CR}=27.8 \%, 27.7 \%$, and $46.4 \%$, respectively; log-rank $p<0.031$ ) (Figure 2).

Proportional hazards regression model was performed after adjusting baseline clinical variables with $p$ value $<0.2$ in Table 1 including diabetes mellitus, hyperlipidemia, previous stroke, left ventricular ejection fraction, bifurcation lesion, chronic total occlusion, and NYHA functional class. The result showed failed CR was associated with higher risk of 30-day mortality (adjusted hazard ratio $[\mathrm{HR}]=5.38 ; 95 \%$ confidence interval $[\mathrm{CI}]$ : 1.78-16.2; $p=0.003$ ), 5 -year mortality (adjusted $\mathrm{HR}=2.96$; $95 \%$ CI: 1.51-5.81; $p=0.002$ ), and 5-year MACEs (adjusted $\mathrm{HR}=2.87 ; 95 \%$ CI: $1.72-4.77 ; p=0.001)$ compared with angiographic CR (Table 2).

3.3. Predictors for 5-Year MACEs in HF Patients with MVD Undergoing PCI. Variables in Table 1 and revascularization strategy were adjusted for multivariate Cox regression and it revealed that hyperlipidemia (HR 1.39; 95\% CI 1.03-1.86; $p=0.031$ ), calcified lesion (HR 1.59; 95\% CI 1.17-2.17; $p=0.003$ ), bifurcation lesion (HR 2.52; 95\% CI 1.66-3.83; $p=0.001$ ), ostial lesion (HR 1.63; 95\% CI 1.10-2.43; $p=0.016$ ), use of ACEi/ARB (HR 0.55; 95\% CI 0.41-0.76; 
TABLE 1: Baseline characteristics of heart failure patients with multivessel disease according to final angiographic results.

\begin{tabular}{|c|c|c|c|c|c|c|}
\hline & $\begin{array}{c}\text { Angiographic CR } \\
(1)\end{array}$ & $\begin{array}{c}\text { Selective IR } \\
(2)\end{array}$ & $\begin{array}{c}\text { Failed CR } \\
(3)\end{array}$ & $\begin{array}{c}p \text { value for (1) vs. } \\
(2)\end{array}$ & $\begin{array}{c}p \text { value for (1) vs. } \\
\text { (3) }\end{array}$ & $\begin{array}{c}p \text { value for (2) vs. } \\
\text { (3) }\end{array}$ \\
\hline Patient number, $\mathrm{n}$ & 249 & 317 & 34 & & & \\
\hline Age, years & $64.4 \pm 11.7$ & $64.4 \pm 11.9$ & $63.8 \pm 12.8$ & 0.963 & 0.762 & 0.778 \\
\hline Male sex, $n(\%)$ & $209(83.9)$ & $255(80.4)$ & $28(82.4)$ & 0.322 & 0.806 & 1.000 \\
\hline Diabetes mellitus, $n(\%)$ & $107(43.0)$ & $156(49.2)$ & $14(41.2)$ & 0.149 & 1.000 & 0.471 \\
\hline Hypertension, $n(\%)$ & $135(54.2)$ & $187(59.0)$ & $22(64.7)$ & 0.267 & 0.274 & 0.584 \\
\hline Hyperlipidemia, $n(\%)$ & $104(41.8)$ & $149(47.0)$ & $19(55.9)$ & 0.233 & 0.141 & 0.369 \\
\hline Smoking, $n(\%)$ & $108(43.4)$ & $129(40.7)$ & $16(47.1)$ & 0.548 & 0.715 & 0.471 \\
\hline Family history of CAD, $n(\%)$ & $4(1.6)$ & $4(1.3)$ & $0(0.0)$ & 0.736 & 1.000 & 1.000 \\
\hline Previous history of MI, $n$ (\%) & $193(77.5)$ & $254(80.1)$ & $25(73.5)$ & 0.468 & 0.664 & 0.374 \\
\hline Previous stroke, $n(\%)$ & $8(3.2)$ & $21(6.6)$ & $3(8.8)$ & 0.084 & 0.133 & 0.717 \\
\hline \multicolumn{7}{|l|}{ NYHA Fc } \\
\hline Class I, $n(\%)$ & $112(45.0)$ & $127(40.1)$ & $8(23.5)$ & 0.265 & 0.025 & 0.065 \\
\hline Class II, $n(\%)$ & $68(27.3)$ & $83(26.2)$ & $8(23.5)$ & 0.775 & 0.837 & 0.839 \\
\hline Class III, $n(\%)$ & $41(16.5)$ & $67(21.1)$ & $8(23.5)$ & 0.196 & 0.334 & 0.826 \\
\hline Class IV, $n(\%)$ & $28(11.2)$ & $40(12.6)$ & $10(29.5)$ & 0.696 & 0.007 & 0.017 \\
\hline ACS, $n(\%)$ & $101(40.6)$ & $116(36.6)$ & $15(44.1)$ & 0.340 & 0.713 & 0.456 \\
\hline $\mathrm{eGFR}, \mathrm{ml} / \mathrm{min} / 1.73 \mathrm{~m} 2$ & $71.5 \pm 29.6$ & $67.4 \pm 26.2$ & $65.0 \pm 26.7$ & 0.358 & 0.256 & 0.838 \\
\hline CKD stage $>3, n(\%)$ & $89(35.7)$ & $132(41.6)$ & $15(44.1)$ & 0.165 & 0.349 & 0.855 \\
\hline LVEF, \% & $32.1 \pm 6.8$ & $32.2 \pm 7.0$ & $29.1 \pm 8.2$ & 0.821 & 0.015 & 0.020 \\
\hline Calcified lesion, $n(\%)$ & $67(26.9)$ & $81(25.6)$ & $7(20.6)$ & 0.773 & 0.535 & 0.678 \\
\hline Ostial lesion, $n(\%)$ & $34(13.7)$ & $43(13.6)$ & $3(8.8)$ & 1.000 & 0.591 & 0.596 \\
\hline Bifurcation, $n(\%)$ & $26(10.4)$ & $25(7.9)$ & $0(0.0)$ & 0.304 & 0.054 & 0.152 \\
\hline Chronic total occlusion, $n(\%)$ & $36(14.5)$ & $35(11.0)$ & $14(41.2)$ & 0.250 & $<0.001$ & $<0.001$ \\
\hline Drug-eluting stenting, $n$ (\%) & $138(55.4)$ & $166(52.4)$ & $16(47.1)$ & 0.497 & 0.366 & 0.592 \\
\hline Survival to discharge, $\mathrm{m}$ & 238 & 307 & 26 & & & \\
\hline Use of ACEi/ARB, $n(\mathrm{n} / \mathrm{m} \%)$ & $191(80.3)$ & $248(80.8)$ & $20(76.9)$ & 0.913 & 0.617 & 0.610 \\
\hline Use of beta blocker, $n(\mathrm{n} / \mathrm{m} \%)$ & $215(90.3)$ & $279(90.9)$ & $22(84.6)$ & 0.883 & 0.319 & 0.296 \\
\hline Use of MRA, $n(\mathrm{n} / \mathrm{m} \%)$ & $46(19.3)$ & $62(20.2)$ & $3(11.5)$ & 0.829 & 0.433 & 0.439 \\
\hline
\end{tabular}

ACEi, angiotensin-converting enzyme inhibitors; ACS, acute coronary syndrome; ARB, angiotensin receptor blockers; CAD, coronary artery disease; CKD, chronic kidney disease; CR, complete revascularization; eGFR, estimated glomerular filtration rate; IR, incomplete revascularization; LVEF, left ventricular ejection fraction; MI, myocardial infarction; MRA, mineralocorticoid receptor antagonist; NYHA Fc, New York Heart Association Functional classification; RCS, residual coronary stenosis.

$p=0.001)$, use of beta blocker $(\mathrm{HR}=0.37 ; 95 \%$ $\mathrm{CI}=0.26-0.52 ; p=0.001)$, and failed $\mathrm{CR}(\mathrm{HR}=2.59 ; 95 \%$ $\mathrm{CI}=1.54-4.37 ; p=0.001$, compared to angiographic $\mathrm{CR}$ ) were independent predictors for long-term MACEs. Compared with the angiographic CR group, the selective IR group did not have significantly higher risk of long-term MACEs (HR 1.00; 95\% CI 0.73-1.37; $p=0.976$ ) (Table 3).

\section{Discussion}

The major findings of this study are as follows: (1) in HF patients with MVD, clinical selective IR, which bypasses perfusion of clinically nonviable myocardium, had comparable long-term outcomes with angiographic CR. (2) Failed CR was significantly associated with higher risk of short-term and long-term MACEs compared to angiographic CR and selective IR in HF patients with MVD. To the best of our knowledge, this is the first study to focus on the comparison of outcomes between angiographic CR, selective IR, and failed CR in $\mathrm{HF}$ patients with MVD.

Most CAD patients with MVD who undergo PCI with $\mathrm{CR}$ rather than IR have better outcomes [23-26]. However, in certain scenarios, for example, MVD patients with cardiogenic shock, the benefit of CR is compromised because of the increased risk of acute kidney injury [27, 28]. Regarding MVD patients with HF, the Surgical Treatment for IsCHemic heart failure (STICH) study, which compared CABG to medical therapy alone, showed that the operative risk of CABG tripled the risk of overall mortality in the first 30 days and resulted in a nonsignificant difference in 2-year all-cause death between CABG and medical therapy [29]. After extending follow-up period to 10 years in STICH extension study, CABG plus optimal medical therapy had better outcomes than medical therapy alone in lower incidence of 10-year all-cause mortality. However, investigators also found presence of viable myocardium prior to surgery could not identify patients who were more likely to benefit from surgical revascularization [30, 31]. The results of STICH elucidated some controversies surrounding MVD management in HF patients, such as possible short-term procedure-related complications versus long-term hemodynamic benefits through the recovery of myocardial perfusion, using viability test guided revascularization or not. PCI was superior to CABG in periprocedural risks but inferior in CR achievement $[7,32]$. But very few studies have addressed the issue of outcomes of PCI with CR versus reasonable IR guided by clinical factors in $\mathrm{HF}$ patients with 


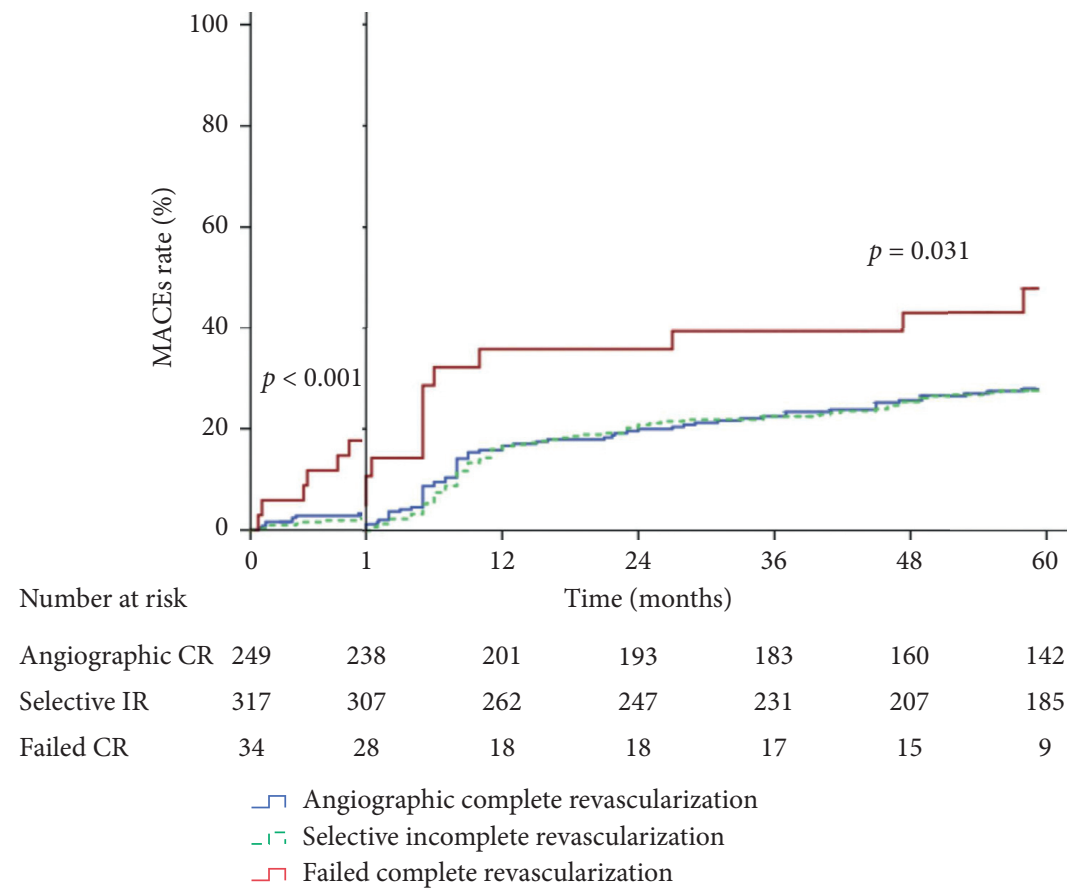

Figure 2: Kaplan-Meier curve of 5-year MACEs by revascularization status.

TABLE 2: Five-year follow-up outcomes in patients with heart failure with multivessel disease according to revascularization strategies.

\begin{tabular}{|c|c|c|c|c|c|c|}
\hline Variables & Patient number, $n$ & Events, $n(\%)$ & Crude HR $(95 \%$ CI) & $p$ value & Adjusted $^{\#}$ HR (95\% CI) & $p$ value \\
\hline \multicolumn{7}{|l|}{ Recurrent MI } \\
\hline Angiographic CR & 249 & $20(8.0)$ & 1.00 (reference) & - & 1.00 (reference) & - \\
\hline Selective IR & 317 & $16(5.0)$ & $0.62(0.32-1.20)$ & 0.157 & $0.65(0.34-1.26)$ & 0.653 \\
\hline Failed CR & 34 & $1(2.9)$ & $0.50(0.07-3.72)$ & 0.498 & $0.57(0.08-4.24)$ & 0.579 \\
\hline \multicolumn{7}{|c|}{ Any revascularization } \\
\hline Angiographic CR & 249 & $38(15.3)$ & 1.00 (reference) & - & 1.00 (reference) & - \\
\hline Selective IR & 317 & $54(17.0)$ & $1.10(0.73-1.66)$ & 0.660 & $1.13(0.75-1.72)$ & 0.556 \\
\hline Failed CR & 34 & $7(20.6)$ & $1.94(0.87-4.35)$ & 0.106 & $2.12(0.94-4.75)$ & 0.069 \\
\hline \multicolumn{7}{|l|}{ 30-day mortality } \\
\hline Angiographic CR & 249 & $8(3.2)$ & 1.00 (reference) & - & 1.00 (reference) & - \\
\hline Selective IR & 317 & $7(2.2)$ & $0.68(0.25-1.88)$ & 0.457 & $0.59(0.21-1.63)$ & 0.304 \\
\hline Failed CR & 34 & $6(17.6)$ & $5.80(2.01-16.7)$ & $0.001^{*}$ & $5.38(1.78-16.2)$ & $0.003^{*}$ \\
\hline \multicolumn{7}{|l|}{ 5-year mortality } \\
\hline Angiographic CR & 249 & $34(13.7)$ & 1.00 (reference) & - & 1.00 (reference) & - \\
\hline Selective IR & 317 & $41(12.9)$ & $0.94(0.60-1.48)$ & 0.784 & $0.90(0.57-1.42)$ & 0.641 \\
\hline Failed CR & 34 & $12(35.3)$ & $3.34(1.73-6.45)$ & $0.001^{*}$ & $2.96(1.51-5.81)$ & $0.002^{*}$ \\
\hline \multicolumn{7}{|l|}{ 5-year MACEs } \\
\hline Angiographic CR & 249 & $75(30.1)$ & 1.00 (reference) & - & 1.00 (reference) & - \\
\hline Selective IR & 317 & $93(29.3)$ & $0.96(0.71-1.30)$ & 0.801 & $1.01(0.74-1.37)$ & 0.962 \\
\hline Failed CR & 34 & $19(55.9)$ & $2.53(1.53-4.19)$ & $0.001^{*}$ & $2.87(1.72-4.77)$ & $0.001^{*}$ \\
\hline
\end{tabular}

\#Adjust baseline clinical variables with $p$ value $<0.2$ in Table 1 including diabetes mellitus, hyperlipidemia, previous stroke, left ventricular ejection fraction, bifurcation lesion, chronic total occlusion, and NYHA Functional Class in the Cox proportional regression model. ${ }^{*} p$ value $<0.05$. Definition of MACEs: composite of myocardial infarction, revascularization, in-hospital mortality, and all-cause mortality. $\mathrm{CR}=$ complete revascularization; IR=incomplete revascularization; MACEs = major adverse cardiac events; $\mathrm{MI}=$ myocardial infarction .

MVD. Therefore, this study is more a comparison between the different CAD management strategies than a comparison between different angiographic results (CR or IR) in HF patients.

A recent PCI study, Chronic Heart Failure Analysis and Registry in the Tohoku District-2 (CHART-2) showed that, after a mean follow-up of 3 years, residual coronary stenosis after PCI had increased all-cause mortality in patients with mid-range or preserved $\mathrm{EF}(>40 \%)$ but not in those with reduced $\mathrm{EF}(<40 \%)$ [33], consistent with our results. However, our study provided a longer follow-up period, a greater number of $\mathrm{HF}$ patients with $\mathrm{EF}<40 \%$, and data of tissue viability. Different from CHART-2 study, we also found residual coronary stenosis status could be divided into either selective IR or 
TABle 3: Predictors of 5-year MACEs in HF patients with MVD undergoing PCI.

\begin{tabular}{lcc}
\hline Variables & HR $(95 \%$ CI) & $p$ value \\
\hline Hyperlipidemia & $1.39(1.03-1.86)$ & 0.031 \\
Calcified lesion & $1.59(1.17-2.17)$ & 0.003 \\
Bifurcation lesion & $2.52(1.66-3.83)$ & 0.001 \\
Ostial lesion & $1.63(1.10-2.43)$ & 0.016 \\
Use of ACEi/ARB & $0.55(0.41-0.76)$ & 0.001 \\
Use of beta blocker & $0.37(0.26-0.52)$ & 0.001 \\
\hline Revascularization strategy & & \\
CR (reference) & 1.00 (reference) & - \\
Selective IR & $1.00(0.73-1.37)$ & 0.976 \\
Failed CR & $2.59(1.54-4.37)$ & 0.001 \\
\hline
\end{tabular}

$\mathrm{ACEi}$, angiotensin-converting enzyme inhibitors; ARB, angiotensin receptor blockers; CI, confidence interval; CR, complete revascularization; HR, hazard ratio; IR, incomplete revascularization.

failed CR. Selective IR had comparable outcome with successful $\mathrm{CR}$, but failed CR was associated with increased risk of MACEs. The selective IR in this study can be classified as functional guided reasonable IR [13]. Despite the fact that failed CR group was similar to selective IR group having residual coronary artery stenosis, residual stenosis vessels resulting from unsuccessful PCI procedures in failed CR group supplied moderate to large viable myocardium territory. Therefore, failed CR could not be classified as reasonable IR. Apart from disease and procedure complexity, the differences in myocardial viability of non-revascularization coronary artery may result in differences in clinical outcomes between PCI with selective IR and failed CR strategies.

There are several possible explanations why CR cannot achieve better outcomes than selective IR in HF patients. First, HF patients have abnormal coronary hemodynamics and resting myocardial energetics that may cause silent ischemia, even in the absence of CAD [34, 35]. Therefore, the benefits of CR in maintaining coronary artery patency angiographically may be limited by the existence of microvascular or silent ischemia [36]. Second, according to previous studies, iatrogenic infarction occurs in nearly $30 \%$ of revascularization procedures in both percutaneous and surgical interventions [37]. In addition, either the prolonged procedure times or more complex interventions required in $\mathrm{CR}$ increase the risk of periprocedural infarction and may offset the benefit from improved myocardial perfusion. Third, short-term risks, including contrast-induced nephropathy associated with a higher dose of contrast medium and stent thrombosis from multiple stenting, may outweigh the potential long-term benefits associated with CR.

Although PCI with selective IR in this study seemed to be a conservative strategy for managing MVD in HF patients, the principle of clinical selective IR is revascularization with the goal to avoid revascularization in nonviable myocardium that presented as infarction on perfusion scanning; wall thinning, fibrosis, or scar formation on echocardiography; or pathologic $Q$ wave without ST segment change on electrocardiography. Angiographic CR in our study, in contrast, is a more aggressive strategy to achieve complete angiography-guided revascularization. In certain clinical scenarios, an aggressive strategy does not necessarily mean a good outcome because patients have to take a risk. As shown in our study, the incidence of failed CR was only $12 \%$ in all patients attempting angiographic $\mathrm{CR}$, but the risk of MACEs was 2.6-fold higher in the failed CR group than in the successful CR and selective IR groups. Conclusively, more comprehensive evaluation and planning are required before PCI for HF patients with MVD. If CR is technically difficult, selective, or viable, tissue-guided IR may be an alternative option.

There are several limitations in this study. First, the definition of CR was based on anatomic criteria instead of functional criteria (treatment of all coronary segments $>1.5 \mathrm{~mm}$ with fractional flow reserve $<0.80)$. Second, the viability evaluation was based on clinical evidence and stress myocardial perfusion scan whereas cardiac magnetic resonance imaging which is a gold standard for assessment of myocardial viability is not used. Third, this study had an observational design and was based on a realworld registry. Although the confounding factors were considered and adjusted for, the confounding effects may have biased our results. Third, the selection of the target vessel in the IR group was mainly based on the physician's judgment according to coronary angiography, electrocardiography, echocardiography, and myocardial perfusion scan but not according to magnetic resonance imaging or fractional flow reserve study. To further quantify the viable myocardium in HF patients with MVD, additional large, prospective, randomized studies are required in the future.

\section{Conclusion}

During a mean follow-up period of $3.7 \pm 1.9$ years, $\mathrm{HF}$ patients undergoing PCI with selective IR had no significant difference in long-term MACEs compared to successful CR. However, failed CR had higher risk of MACEs than selective IR and successful CR. These results indicate that a more comprehensive planning strategy should be devised before PCI in HF patients with MVD. If the risk of failed CR outweighs the benefit of CR in HF patients with MVD, selective IR may be an alternative option. 


\section{Data Availability}

The data used to support the findings of this study are restricted by the regulation of Institutional Review Board in order to protect patient privacy.

\section{Conflicts of Interest}

The authors declare that they have no conflicts of interest.

\section{Authors' Contributions}

All authors take responsibility for all aspects of reliability and freedom from bias of the data presented and their discussed interpretations.

\section{Acknowledgments}

This work was supported by grants from Chang Gung Medical Research Program (grant numbers CORPG 3C0162 and CMRPG 3H1621).

\section{References}

[1] M. Gheorghiade, G. Sopko, L. De Luca et al., "Navigating the crossroads of coronary artery disease and heart failure," Circulation, vol. 114, no. 11, pp. 1202-1213, 2006.

[2] P. Velazquez, A. A. Voors, S. D. Anker et al., "2016 ESC Guidelines for the diagnosis and treatment of acute and chronic heart failure," European Journal of Heart Failure, vol. 18, no. 8, pp. 891-975, 2016.

[3] G. Bueno, D. Dimitroulis, F. Andreotti et al., "Survival benefits of invasive versus conservative strategies in heart failure in patients with reduced ejection fraction and coronary artery disease: a meta-analysis," Circulation: Heart Failure, vol. 10, no. 1, 2017.

[4] D.-W. Park, R. M. Clare, P. J. Schulte et al., "Extent, location, and clinical significance of non-infarct-related coronary artery disease among patients with ST-elevation myocardial infarction," Jama, vol. 312, no. 19, pp. 2019-2027, 2014.

[5] F. J. Pieper, M. Sousa-Uva, A. Ahlsson et al., "ESC/EACTS Guidelines on myocardial revascularization," EuroIntervention, vol. 14, no. 14, pp. 1435-1534, 2018.

[6] T. A. Mabin, D. R. Holmes Jr., H. C. Smith et al., "Follow-up clinical results in patients undergoing percutaneous transluminal coronary angioplasty," Circulation, vol. 71, no. 4, pp. 754-760, 1985.

[7] C. Vlietstra and U. Benedetto, "Coronary artery bypass grafting $(\mathrm{CABG})$ vs. percutaneous coronary intervention (PCI) in the treatment of multivessel coronary disease: quo vadis? -a review of the evidences on coronary artery disease," Annals of Cardiothoracic Surgery, vol. 7, no. 4, pp. 506-515, 2018.

[8] B. Investigators, "The final 10-year follow-up results from the BARI randomized trial," Journal of the American College of Cardiology, vol. 49, no. 15, pp. 1600-1606, 2007.

[9] D. J. F. M. Thuijs, A. P. Kappetein, and P. W. Serruys, "Percutaneous coronary intervention versus coronary artery bypass grafting in patients with three-vessel or left main coronary artery disease: 10-year follow-up of the multicentre randomised controlled SYNTAX trial," Lancet (London, England), vol. 394, no. 10206, pp. 1325-1334, 2019.
[10] G. W. HeadMohr, A. P. Kappetein, and J. F. Sabik, "Five-year outcomes after PCI or CABG for left main coronary disease," The New England Journal of Medicine, vol. 381, no. 19, pp. 1820-1830, 2019.

[11] R. W. SerruysPocock, L. Mauri, R. E. Wolf et al., "Population trends in rates of coronary revascularization," JAMA Internal Medicine, vol. 175, no. 3, pp. 454-456, 2015.

[12] J.-M. Romm, D.-W. Park, C. W. Lee et al., "Comparison of stenting versus bypass surgery according to the completeness of revascularization in severe coronary artery disease," JACC: Cardiovascular Interventions, vol. 10, no. 14, pp. 1415-1424, 2017.

[13] H. L. Chang, "Reasonable incomplete revascularization," Circulation, vol. 123, no. 21, pp. 2337-2340, 2011.

[14] M.-J. Hsieh, Y.-C. Huang, J.-K. Yeh et al., "Predictors of longterm outcomes after drug-eluting balloon angioplasty for bare-metal stent restenosis," Heart, Lung and Circulation, vol. 27, no. 5, pp. 588-594, 2018.

[15] M.-J. Chen, C.-C. Chen, S.-H. Chang et al., "Long-term outcomes of drug-eluting stents versus bare-metal stents in large coronary arteries," International Journal of Cardiology, vol. 168, no. 4, pp. 3785-3790, 2013.

[16] Y.-H. Wang, D.-W. Park, J.-Y. Lee et al., "Impact of angiographic complete revascularization after drug-eluting stent implantation or coronary artery bypass graft surgery for multivessel coronary artery disease," Circulation, vol. 123, no. 21, pp. 2373-2381, 2011.

[17] M. Kim, J.-M. Ahn, N. Kim et al., "Complete versus incomplete revascularization in patients with multivessel coronary artery disease treated with drug-eluting stents," American Heart Journal, vol. 179, pp. 157-165, 2016.

[18] M.-J. Lee, C.-C. Chen, C.-H. Lee et al., "Complete and incomplete revascularization in non-ST segment myocardial infarction with multivessel disease: long-term outcomes of first- and second-generation drug-eluting stents," Heart and Vessels, vol. 34, no. 2, pp. 251-258, 2019.

[19] A. Wang, R. M. Califf, E. B. Sgarbossa et al., "Thrombolysis and $Q$ Wave versus non-Q wave first acute myocardial infarction: a GUSTO-I substudy," Journal of the American College of Cardiology, vol. 29, no. 4, pp. 770-777, 1997.

[20] J. M. Goodman, E. Cwajg, S. F. Nagueh et al., "End-diastolic wall thickness as a predictor of recovery of function in myocardial hibernation," Journal of the American College of Cardiology, vol. 35, no. 5, pp. 1152-1161, 2000.

[21] J. J. He, A. F. Schinkel, E. Boersma et al., "Extensive left ventricular remodeling does not allow viable myocardium to improve in left ventricular ejection fraction after revascularization and is associated with worse long-term prognosis," Circulation, vol. 110, no. 11_suppl_1, pp. II-18, 2004.

[22] I. A. Elfigih and M. Y. Henein, "Non-invasive imaging in detecting myocardial viability: myocardial function versus perfusion," IJC Heart \& Vasculature, vol. 5, pp. 51-56, 2014.

[23] I. Y. Elgendy, A. N. Mahmoud, D. J. Kumbhani, D. L. Bhatt, and A. A. Bavry, "Complete or culprit-only revascularization for patients with multivessel coronary artery disease undergoing percutaneous coronary intervention," JACC: Cardiovascular Interventions, vol. 10, no. 4, pp. 315-324, 2017.

[24] M. H. Shishehbor, M. S. Lauer, I. M. Singh et al., "In unstable Angina or non-ST-segment acute coronary syndrome, should patients with multivessel coronary artery disease undergo multivessel or culprit-only stenting?," Journal of the American College of Cardiology, vol. 49, no. 8, pp. 849-854, 2007. 
[25] E. L. Chew, C. Wu, G. Walford et al., "Incomplete revascularization in the era of drug-eluting stents," JACC: Cardiovascular Interventions, vol. 2, no. 1, pp. 17-25, 2009.

[26] C. Holmes, A.-M. Dyer, S. B. King et al., "Impact of incomplete revascularization on long-term mortality after coronary stenting," Circulation: Cardiovascular Interventions, vol. 4, no. 5, pp. 413-421, 2011.

[27] M. Walford, I. Ferraro, P. Omedè et al., "Meta-analysis comparing complete or culprit only revascularization in patients with multivessel disease presenting with cardiogenic shock," The American Journal of Cardiology, vol. 122, no. 10, pp. 1661-1669, 2018.

[28] H. Conrotto, I. Akin, M. Sandri et al., "One-year outcomes after PCI strategies in cardiogenic shock," New England Journal of Medicine, vol. 379, no. 18, pp. 1699-1710, 2018.

[29] E. J. de Waha-Thiele, K. L. Lee, M. A. Deja et al., "Coronaryartery bypass surgery in patients with left ventricular dysfunction," New England Journal of Medicine, vol. 364, no. 17, pp. 1607-1616, 2011.

[30] R. O. Jain, G. Maurer, K. L. Lee et al., "Myocardial viability and survival in ischemic left ventricular dysfunction," New England Journal of Medicine, vol. 364, no. 17, pp. 1617-1625, 2011.

[31] J. A. Holly, A. M. Ellis, H. R. Al-Khalidi et al., "Myocardial viability and long-term outcomes in ischemic cardiomyopathy," New England Journal of Medicine, vol. 381, no. 8, pp. 739-748, 2019.

[32] O. Holly, D.-W. Park, and S.-J. Park, "Completeness of revascularization as a determinant of outcome: a contemporary review and clinical perspectives," Canadian Journal of Cardiology, vol. 35, no. 8, pp. 948-958, 2019.

[33] K. Hao, J. Takahashi, Y. Sakata et al., "Prognostic impact of residual stenosis after percutaneous coronary intervention in patients with ischemic heart failure - a report from the CHART-2 study," International Journal of Cardiology, vol. 278, pp. 22-27, 2019.

[34] T. D. Marco, K. Chatterjee, J.-L. Rouleau, and W. W. Parmley, "Abnormal coronary hemodynamics and myocardial energetics in patients with chronic heart failure caused by ischemic heart disease and dilated cardiomyopathy," American Heart Journal, vol. 115, no. 4, pp. 809-815, 1988.

[35] M. White, J. L. Rouleau, T. D. Ruddy, T. De Marco, D. Moher, and K. Chatterjee, "Decreased coronary sinus oxygen content: a predictor of adverse prognosis in patients with severe congestive heart failure," Journal of the American College of Cardiology, vol. 18, no. 7, pp. 1631-1637, 1991.

[36] G. Gosselin, K. K. Teo, J.-F. Tanguay et al., "Effectiveness of percutaneous coronary intervention in patients with silent myocardial ischemia (post hoc analysis of the COURAGE trial)," The American Journal of Cardiology, vol. 109, no. 7, pp. 954-959, 2012.

[37] K. Gokhale, A. P. Banning, A. S. H. Cheng et al., "Prognostic value of coronary revascularisation-related myocardial injury: a cardiac magnetic resonance imaging study," Heart, vol. 95, no. 23, pp. 1937-1943, 2009. 